\title{
CULTURAL HERITAGE AND INTEROPERABLE OPEN PLATFORMS: STRATEGIES FOR KNOWLEDGE, ACCESSIBILITY, ENHANCEMENT AND NETWORKING
}

\author{
DANILA LONGO*, ANDREA BOERI $\dagger$, BEATRICE TURILLAZZI $†$ \& SERENA ORLANDI§ \\ Department of Architecture, University of Bologna, Italy
}

\begin{abstract}
Improving the accessibility of information on cultural heritage is a key step to achieve sustainable and inclusive valorisation actions recognising knowledge as a valuable and operable resource. This paper addresses the issue from the point of view of digital technologies, in particular interoperable digital platforms for the storage, processing and visualization of open and interoperable data available in different formats (3D models, images, texts, etc.) and collected from different sources (traditional archives, digital libraries, real-time content, IoT, sensors, social networks, etc.). The comparison among three projects with different focuses - the H2020 INCEPTION project (Inclusive Cultural Heritage in Europe through 3D semantic Modelling - G.A. No. 665220), the H2020 ROCK project (Regeneration and Optimization of Cultural heritage in creative and Knowledge - G.A. No. 730280) and the Italian national project PON PNR IDEHA (Innovation for Data Elaboration in Heritage Areas - No. ARS01_00421) - shows possible strategies led by the knowledge of data usage models and information databases, focused on the common goal of improving accessibility, knowledge, enhancement and conservation of cultural heritage. The results show how information management through open and interoperable platforms represents a useful decision and monitoring support system related to cultural heritage: further, the provision of data and digital sources to different users, the use of this tool can create collaborative networks between all stakeholders involved at various levels and to allow new potential knowledge scenarios.
\end{abstract}

Keywords: cultural heritage, interoperable platforms, open data, digital resources, digital information systems, accessibility, enabling technologies.

\section{INTRODUCTION}

The term "platform" generically indicates a space with a flat surface and it can be referenced to different meanings: it is a structure that has a horizontal, fixed or mobile articulation, which works as a base, support or connection in building construction or mechanics; it is the plant intended for drilling wells, extraction and transport of raw materials such as hydrocarbons in the extraction industry; it is the combination of technologies that control a digital system and manage its access procedures; in IT language, it is the processing base consisting of the hardware and operating system of a computer on which programs and applications are developed and run [1]; it is the space of interaction between users connected to digital social networks through a form of contextual communication; and many others.

The common condition to the different fields of use of a platform is its open and democratic role of collection, support, extraction, exchange, connection, interaction and "social" relationship between the resources and their users: this horizontal and participative way makes it a useful tool with great potential in the field of cultural heritage $(\mathrm{CH})$, that is by definition, a resource of common interest and universal value.

*ORCID: https://orcid.org/0000-0002-7516-7556

†ORCID: https://orcid.org/0000-0003-1390-2030

†ORCID: https://orcid.org/0000-0001-5864-2055

${ }^{\S}$ ORCID: https://orcid.org/0000-0003-0868-3080 
The UNESCO Convention concerning the Protection of the World Cultural and Natural Heritage (1972) describes in detail the large and diverse extent of this Heritage: "Monuments [...] which are of outstanding universal value from the point of view of history, art or science; Groups of buildings [...] of outstanding universal value from the point of view of history, art or science; Sites $[\ldots]$ which are of outstanding universal value from the historical, aesthetic, ethnological or anthropological point of view; Natural features [...] which are of outstanding universal value from the aesthetic or scientific point of view; Geological and physiographical formations [...] of outstanding universal value from the point of view of science or conservation; Natural sites [...] of outstanding universal value from the point of view of science, conservation or natural beauty". The Convention also recognizes that the duty of ensuring the identification, protection, conservation, presentation and transmission to future generations of the cultural and natural heritage belongs primarily to that State in which territory it is situated [2].

The Italian legislation clarifies how the cultural and natural heritage ("immovable and movable assets presenting artistic, historical, archaeological, ethno-anthropological, archival and bibliographical interest as example of civilisation; buildings and sites representing the expression of the historical, cultural, natural, morphological and aesthetic values of the territory") are intended for the fruition of the community and how actions aiming at its protection and valorisation contribute to promote the development of culture and preserve the memory of the national identity and its territory [3].

Besides the "tangible heritage", this "memory" has been broadened to the immaterial dimension of the knowledge arising from the development and experience of human practices and related spatial, social and cultural constructions [4] - "practices, representations, expressions, knowledge, skills, as well as the instruments, objects, artefacts and cultural spaces associated therewith, transmitted from generation to generation by communities, groups and individuals as evidence of identity and continuity" [5] - as ratified since the 2003 Paris UNESCO Convention.

In the Council of Europe Framework Convention on the Value of Cultural Heritage for the Society signed in Faro in 2005, the "universal" value of $\mathrm{CH}$ is turned into "individual and collective responsibility" supported by the acknowledgement of participation in cultural life as a right, equal to those stated in the Universal Declaration of Human Rights: $\mathrm{CH}$ takes an active role in the construction of a peaceful and democratic society, in the processes of sustainable development, in the promotion of cultural diversity and for the enhancement of the synergy of competences among all the public, institutional and private actors concerned. In the spirit of democratic participation, law and responsibility must be based on $\mathrm{CH}$ knowledge and accessibility: an action to be developed also thanks to digital technologies (Art. 14 - Cultural heritage and the information society), through the promotion of high quality contents, the definition of internationally compatible standards, the removal of obstacles to access to information (particularly for educational purposes), whilst protecting intellectual property rights and ensuring that the creation of digital content does not prejudice the conservation of existing heritage [6].

Therefore, the collective and community approach characterising the $\mathrm{CH}$ finds in the social and networked space of the interoperable digital platform a perfect place to implement common responsibility for the protection, enhancement and promotion of memory and common legacy. This action occurs through strategies able to enhance $\mathrm{CH}$ in terms of knowledge, accessibility to resources and creation of a collaborative and sharing network and these become useful tools for innovative and effective inclusive policies and initiatives "of sustainable development and quality of life in an ever-changing society" [6], to be used in 
many scenarios related to the social, culture, the economy, urban regeneration policies, tourism, etc.

\section{DIGITAL INFORMATION IN THE INFORMATION SOCIETY}

In the modern information society, information and communication technologies (ICTs) enable a large amount of data to be accessed, processed, transferred and stored, and these data are now crucial for social, economic, political and cultural life at global level. The process of digitisation, transmission and storage of information, which has been growing steadily since 2002 (when for the first time in human history the amount of information stored on digital devices exceeded the amount kept on analogue storage archives), has significantly led to modernisation of education, more transparency, higher economic productivity and less bureaucracy. However, this scenario is accompanied by a surprising lack of awareness of information, access difficulties and unsolved digital divide in the information society [7].

According to the United Nations Conference on Trade and Development (UNCTAD), the global emergency due to COVID-19 shown the impact of digital readiness - the level of digitization and the related opportunity to benefit from digital capabilities - on the adequacy of the response of individual countries to the pandemic [8]. During the lockdown, this condition concerned and enabled the management of essential public administration and health services, the remote transfer of work, commercial or educational activities, the contact tracing test, as well as giving continuity to social relations and leisure opportunities. The potential and key role of ICT in managing the emergency increases the need for the introduction of effective policies, according to a coordinated multilateral and multistakeholder approach, to overcome the long-standing global inequalities affecting people's ability to use the internet and other digital technologies (more than half of the world's population is able to access the internet, while in the LDCs (least developed countries) the proportion increases to one in five) [8]. As outlined by the Secretary-General of UNCTAD and UNGIS Chair, there is an urgent need to pay the appropriate attention to overcoming existing and emerging digital divides by providing a multilateral response coordinated to the issues of digitization deployment, which can also help countries build up their resilience and facilitate solutions to other cross-cutting challenges, such as climate change and the current COVID-19 pandemic [9].

However, the issue of digitisation and the gap between countries already included in the ICT society and those still excluded does not only affect countries with emerging economies. At European level, since December 2006, the European Commission has launched the Digital Skills and Jobs Coalition (DSJC), gathering under the management of Digital Europe (the leading trade association representing digitally transforming industries in Europe) a large group of actors representing a plurality of industries, education and training organisations, social players, NGOs and EU Member States. The common goal is to address the lack of eskills in Europe through concrete actions and by sharing best practice experiences on e-skills development. The activities of the Coalition focus on four main themes: Digital skills for all citizens (skills that enable all citizens to be active in our digital society); Digital skills for the workforce (skills for the digital economy, e.g. skills and retraining); Digital skills for ICT professionals (high-level skills for ICT professionals in all industrial sectors); and Digital skills in education (innovative teaching and learning e-skills) [10]. An example of the implementation of these actions in the Italian context is the National Coalition set up within the "Repubblica Digitale", the national strategic initiative promoted by the Department for Digital Transformation of the President of the Council of Ministers to tackle the digital divide in Italy, to support the greatest digital inclusion and to promote education on the technologies of the future, guiding the process of digital transformation of the country. 
The acknowledgment and the increase of the benefits of information technologies for economic growth, job creation and the quality of life are also part of the Europe 2020 strategy of the Digital Agenda for Europe. A strategy refocusing attention on another important field of opportunities related to the use of the internet: the optimization of the economic and cultural potential of the European $\mathrm{CH}$. Recommendations on the digitisation and online accessibility of cultural material and digital preservation, published in 2006 and later resumed in 2011, emphasize the advantages given by the online accessibility of cultural sources "that will make it possible for citizens throughout Europe to access and use it for leisure, studies or work or it will give Europe's diverse and multilingual heritage a clear profile on the internet, and the digitisation of their assets will help Europe's cultural institutions to continue carrying out their mission of giving access to and preserving our heritage in the digital environment" - and the potential fields of application of digitised data - "that can be reused, for both commercial and non-commercial purposes, for uses such as developing learning and educational content, documentaries, tourism applications, games, animations and design tools". Digitising and providing wider access to cultural resources is considered an essential condition for the further development of Europe's cultural and creative capacities, and of its creative industries landscape [11].

Moreover, according to the New European Agenda for Culture of 2018, besides enabling new and innovative forms of artistic creation, the digital revolution is shaped as new ways to access, consume and monetise cultural content [12].

Actions such as making better use of advanced digital technologies for the protection and the fruition of $\mathrm{CH}$, increasing the involvement of citizens and the spillover support in other sectors, enhancing cross-sector and cross-border cooperation and capacity building in the sector of digitised $\mathrm{CH}$, also return as central objectives of the "Declaration of cooperation on advancing digitisation of Cultural Heritage" signed during the Digital Day 2019. According to the Declaration, the digital transformation can play an essential role in enabling cultural experiences, knowledge creation, preservation, use and re-use of $\mathrm{CH}$ across borders. Digitised cultural objects moreover unlock the potential for broader societal, cohesive and economic benefits of areas such as tourism, education and creative sectors [13].

The ability of ICT to facilitate more open and inclusive access to information (which became particularly evident during the current pandemic emergency) is therefore a fundamental field of action and experimentation for $\mathrm{CH}$ : an open challenge to make more resources available providing opportunities to participate actively, to protect and promote Europe's cultural heritage as a shared common pool resource and to bring tangible benefits to economies and societies.

\section{DIGITAL PLATFORMS FOR CH}

The ecosystem of digital resources related to $\mathrm{CH}$, as well as the assets composing it, is wide and multifaceted. The knowledge basis densely populated by a large amount of information about $\mathrm{CH}$ derives from equally numerous sources: web pages and relevant databases of Community institutions and national states, cultural authorities, cultural heritage institutions and associations or museums; catalogues of libraries, bibliographic directories and online libraries; indexes of archives and online archives; specialized periodicals; search engines; blogs and forums; open dataset databases; open access or open research data repository of scientific results. In order to describe the extensive network of knowledge-holding "sites" on $\mathrm{CH}$, a very long list is needed, matching the more traditional repositories already mentioned with a newly developed framework of services, websites and platforms, representing the preferred systemic access point to information on digital and digitized content of museums, galleries, libraries, archives and cultural institutions, which in some cases include the 
possibility of collaborative contributions, services and additional application tools (i.e. Europeana version pro). Online maps and social media complement this source list. A stateof-the-art mapping of information systems on $\mathrm{CH}$ is now being processed as part of the actions of the PON (National Operational Program) IDEHA project of Industrial research and experimental development.

Notwithstanding the acknowledged strategic role of digital technologies for the community in terms of ability to transfer the memory and the outstanding universal value of $\mathrm{CH}$ (in space, through communication, and in time, through storage, using the digital information produced, stored and processed), it is still difficult to orientate in this apparently uncoordinated system, with no hierarchy and not always based on direct and linear relations.

The importance and role of a European coordination and valorisation activity of national digitisation programmes for cultural information (starting from 2001 with the Lund Charter of Principles and the following Action Plan [14], the already mentioned recommendations 2006/585/EC and 2011/711/EU of the European Commission, the Dynamic Action Plan for the European coordination of digitisation of cultural and scientific content of 2005 [15], until the "Declaration of cooperation on advancing digitisation of cultural heritage" of 2019) is widely recognized and agreed, aiming to ensuring a greater and easy access to and use of cultural content, avoiding data dispersion and duplication of efforts, overlap in digitisation and supporting the deployment and long-term data conservation strategies.

However, this is still an open challenge facing issues - already tackled with the FAIR (Findable, Accessible, Interoperable, Reusable) Data Principles and object of research activities in humanities and social science field - related to fragmentation (the information remains largely disjointed, typically residing with the individuals that produce it rather than in shared or common repositories known to a site manager or international conservation organizations); reliability (related to the growth in the quantity of data produced by high numbers and different backgrounds individuals, labelling or cataloguing errors); longevity of data (digital material has to be managed and maintained during time - without special care, heritage records stand little chance of surviving as long as the heritage they are meant to document) (Addison et al. [16]); availability and accessibility; domain visual protection measures and copyright issues.

In addition to methodological issues related to cataloguing, storage, data correlation or multiple access points, other aspects highlight the sophistication of the $\mathrm{CH}$ information picture:

- data refer to "objects" of different nature ranging from a painting, a monumental complex, a book or collections of books, buildings, up to assets belonging to the intangible heritage;

- as a consequence, data have different consistencies translated into many different descriptive supports (writings, tables, audio, video, images, 2D or 3D graphic elaborations, etc.); exchanged on different formats (tabular: csv, xls, etc.; geographic vector: shp, dwg, etc.; images: jpg, png, etc.; structured data: rdf, xml, etc.; texts: doc, txt, pdf, etc.; audio and video: mp3, mp4) and viewable, sometimes, on dedicated software (HBIM, GIS, CAD, etc.);

- data come from a variety of actors - institutions, researchers, $\mathrm{CH}$ experts, companies, associations, public and cultural institutions, etc. - are produced and stored in different environments - from scientific records to historical studies, surveys, inventories, photos campaigns, maps, field documentation, restoration, monitoring activities, etc. - using as many surveying, diagnostic or conservation devices - in some cases highly technological 
(such as 3D scanners, photogrammetric and surveying instruments, GPS sensors, thermal and acoustic detectors, environmental sensors, etc.);

- there are many different purposes and fields of application of data (study, research, restoration, cultural promotion, tourism, etc.), there are many actors producing this information, there are many end users targeted (citizens in general, students, researchers, tourists, teachers, etc.).

Reminding the Faro Convention, the user and his right to benefit from the transfer of cultural knowledge (supported by ICTs) represent the aim which should guide the actions on increasing the information systems of the $\mathrm{CH}$, according to an inclusive and friendly approach. "Users need to benefit more from the networking of cultural knowledge, as the implementation of technologies enables the development of a European cultural information space. They need to be facilitated to find easily and use cultural content and to contribute their own knowledge and experience, becoming active citizens in information societies" [15]. A "benefit" that goes in many directions: while citizens are now experiencing unique opportunities to access cultural contents, on the other hand institutions can reach out to broader audiences, engage new users and develop creative and accessible content for leisure and education [17] or develop cross-cutting collaboration involving stakeholders from different backgrounds and disciplines. This is a key point showing the high potential value of information systems as tools for engaging stakeholders at various levels and supporting the implementation of effective policies for the valorisation of $\mathrm{CH}$; tools to be used for the growth of other sectors, such as cultural tourism or in urban regeneration processes.

The digital platform, broadly deployed as a social and economic interaction system, is a powerful enabling technology for the exploitation of cultural information and, if interoperable and based on open data, enables continuous communication, cooperation and data exchange with other systems, optimising resources. This process is obtained using the ontology, a system that represents the amount of data and their relationships in a given knowledge domain: a description model that allows to compare data generated and provided by different environments and made available in different formats, fragmented so far, clustered on the basis of their relationships. Books, works of art, documents, archaeological artefacts, digital-born objects, etc. are no longer single entities, becoming nodes in an open and continuously updated network of meanings. The interlinking network of stored data and the opportunity to search by words and, mostly, by means, become an added value. Ontologies, besides creating a tight net of links between information, enable the interoperable platform to be a tool of opportunity in building an even tight ecosystem of relationships among users, stakeholders and resources.

The management and the accessibility of the available wide and valuable databases (digitised or digitally-born) on $\mathrm{CH}$ can have a significant impact on many fields of research and exploitation: a potentiality that finds in the systematization of this asset with the different actors produce, process or use information, the opportunity to build new connections, promoting its use and reuse for the creation of new heritage.

\section{GOOD PRACTICES RELATED TO CH-DRIVEN PLATFORMS: INCEPTION, ROCK AND IDEHA RESEARCH PROJECTS}

A well-designed digital platform can successfully meet the need to communicate, make accessible, exchange and systematise resources and knowledge of $\mathrm{CH}$ (according to its inherent complexity features) when it satisfies a combination of skills and objectives: to preserve knowledge, protecting its value, meaning and integrity; to store data of different origins and formats expressing their multidisciplinary features (from maps to images, 
drawings, documents, CAD files, movies, audio recordings, etc.); to ensure the long-term viability of the records through clear, simple, and human-decodable data management systems; to allow data access and search in an inclusive way by a simple and intuitive querying in order to reach a wide and varied target group of users; to foster participation and enrichment of heritage in terms of additional contributions of records; to promote interest in $\mathrm{CH}$ through the dissemination of acquired information; to enable informed decision making [16]; to serve as a tool for monitoring the impacts of adopted policies; to increase transparency and democratic participation via the use of open and interoperable data; to improve the efficiency and effectiveness of services to citizens through innovative systems; to become a medium of engagement and cross-sector networking between data and stakeholders. These ambitious goals identify the knowledge and the assets of $\mathrm{CH}$ as common, valuable, active and operable affairs and define the digital platform not merely as a valuable support for data collection and visualization but as a working tool for accessibility, knowledge, understanding, enhancement and preservation of the collective heritage.

The common experience developed in three research projects, INCEPTION, ROCK and IDEHA, concerning the creation of interoperable platforms for repository, processing and visualization of open and interoperable data of different format ( $3 \mathrm{~d}$ models, images, texts, etc.), collected from different sources (traditional repositories, digital libraries, real-time contents, IoT, sensors, social networks, etc.) and targeting multiple types of users (researchers, authorities, policy makers, tourists, citizens, etc.), shows possible knowledgedriven strategies of data use models and information databases and datasets, clarifying the potentialities, impacts and results of the interaction between platform and actions on $\mathrm{CH}$. Starting from this common tool, the three projects address the challenge of the digitisation of $\mathrm{CH}$ and the access to the corresponding digitised resources on various areas of application and related to different levels of scale: from historic buildings and urban districts to a wide spectrum of contexts related to $\mathrm{CH}$.

The H2020 EU funded INCEPTION project (Inclusive Cultural Heritage in Europe through 3D semantic modelling, 2015-2019-G.A. No. 665220) deals with the conservation, valorisation and improvement of accessibility to $\mathrm{CH}$ by focusing on $3 \mathrm{D}$ modelling. Using BIM (Building Information Modelling) methods, the project developed a protocol for supporting the production of digital models of buildings and archaeological sites which is able to associate the geometric data (resulting from 3D survey and data capturing techniques such as laser scanner or 3D photogrammetry) to digital data of other origins, through an openstandard and interoperable Semantic Web platform. The platform acquires data related to 3D models, assigns them geo-spatial coordinates (reporting their geographical position on a plan sphere) and enriches them with further useful descriptive information - for example, historical documents or environmental analysis - able to describe their physical characteristics at certain time periods, which can be potentially continuously enriched and implemented (Fig. 1). The open system allows the sharing, access and use of models to researchers from different disciplines and non-expert users, enabling easy interactions through functionalities as Augmented and Virtual Reality, the generation of visualizations by selecting a specific historical moment or a physical characteristic, providing additional reference information. The process has been tested on demonstration cases quite varied (by type, physical size, state of preservation, purpose of digitization, etc.) in order to comply with the complexities of $\mathrm{CH}$. "The technical innovation of the INCEPTION platform redefines the meaning of 3D models of cultural heritage, redefining them as knowledge aggregators" [18] and confirms how the application of semantic-based digital technologies represents a key resource to build an open network of relationships between assets and content (otherwise 


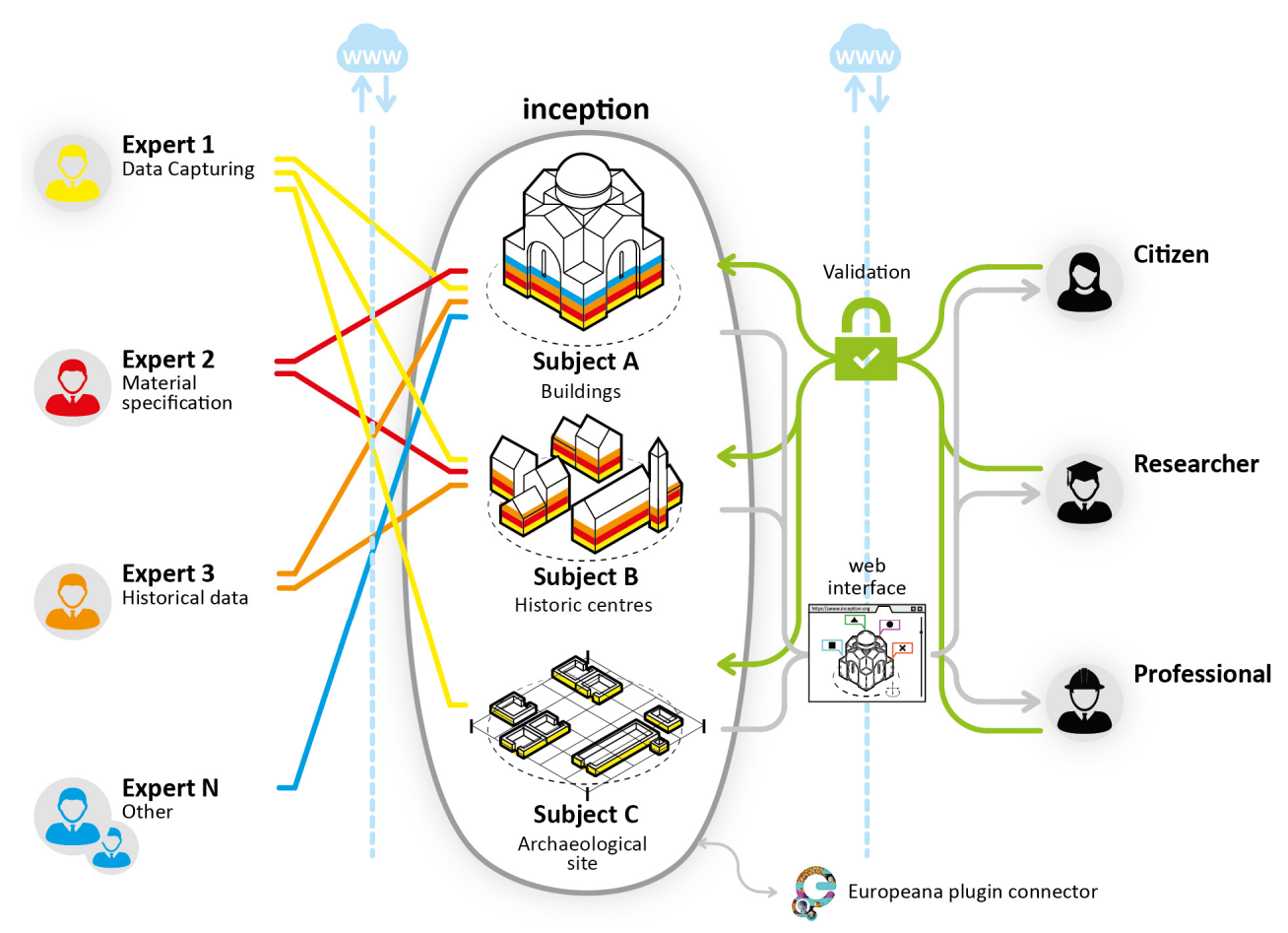

Figure 1: The INCEPTION Stakeholder ecosystem panel [18].

unconnected and connectable), configuring three-dimensional representation as a plan for a more inclusive interdisciplinary exchange [18], [19].

The H2020 EU funded ROCK project (Regeneration and Optimization of Cultural heritage in creative and Knowledge, 2017-2020 - G.A. No. 730280) works on historical city centres as a testing ground for innovative $\mathrm{CH}$-driven regeneration strategies, assumed as an impressive factor of sustainable development and economic growth for cities. The project, funded in 2017 under the H2020 programme in the axis Climate - Greening the Economy, in response to the call "Cultural Heritage as a driver for sustainable growth", is leaded by the Municipality of Bologna supported by the University of Bologna as scientific coordinator and involves 10 European Cities (seven role model cities: Athens, Cluj-Napoca, Eindhoven, Liverpool, Lyon, Turin and Vilnius, where successful processes of regeneration have already implemented and three replicator cities: Bologna, Lisbon and Skopje, where developing similar models in relation to their specific local contexts) for a total of 32 partners. One of the several actions performed in the project is the ROCK Platform, a heterogeneous data sources management and intervention-oriented tool for complex urban systems to store, visualize, process and link data on $\mathrm{CH}$. The starting point is the awareness that, in order to increase the value of $\mathrm{CH}$ and to promote its use, information should be made clearly accessible to all actors involved (institutions, stakeholders, cultural operators, policy makers, scholars, researchers, professionals, curators or merely city users as tourists, workers or residents) to interact with knowledge about $\mathrm{CH}$, to exchange experiences and to provide new data and contents. The multilevel platform is driven by multiple sources, i.e. primary data (acquired from environmental and visitor flow sensors installed in the involved cities and 
collected in an Atlas) and secondary data (data already owned by the municipalities or made available by the curators) and makes the datasets accessible and downloadable as open data. At the same time the platform enables the use of different dashboards which aggregate, compare and report in a synthetic and user-friendly interface the data (through tables, graphs or maps) in order to measure and monitor some reference parameters (data on climate, pollution, noise, flows of people, nationality of visitors), the progress of the actions carried out in the three city replicators and the comparison of the different scenarios (Fig. 2). One of the ROCK innovative contribution to CH ICT field is the development of a semantic contestbased framework based on a participatory methodology (founded on the three main thematic areas: Sustainability, Accessibility and Collaboration for new cultural productions) useful in translating data into the supporting system by means of filters and descriptors that could describe and respond to the needs of heritage-led regeneration actions and strategies implemented by the project in historical complex urban context. In this way, the platform simplifies access to information and enables the exchange among citizens, authorities and entrepreneurs through networking [20], [21].

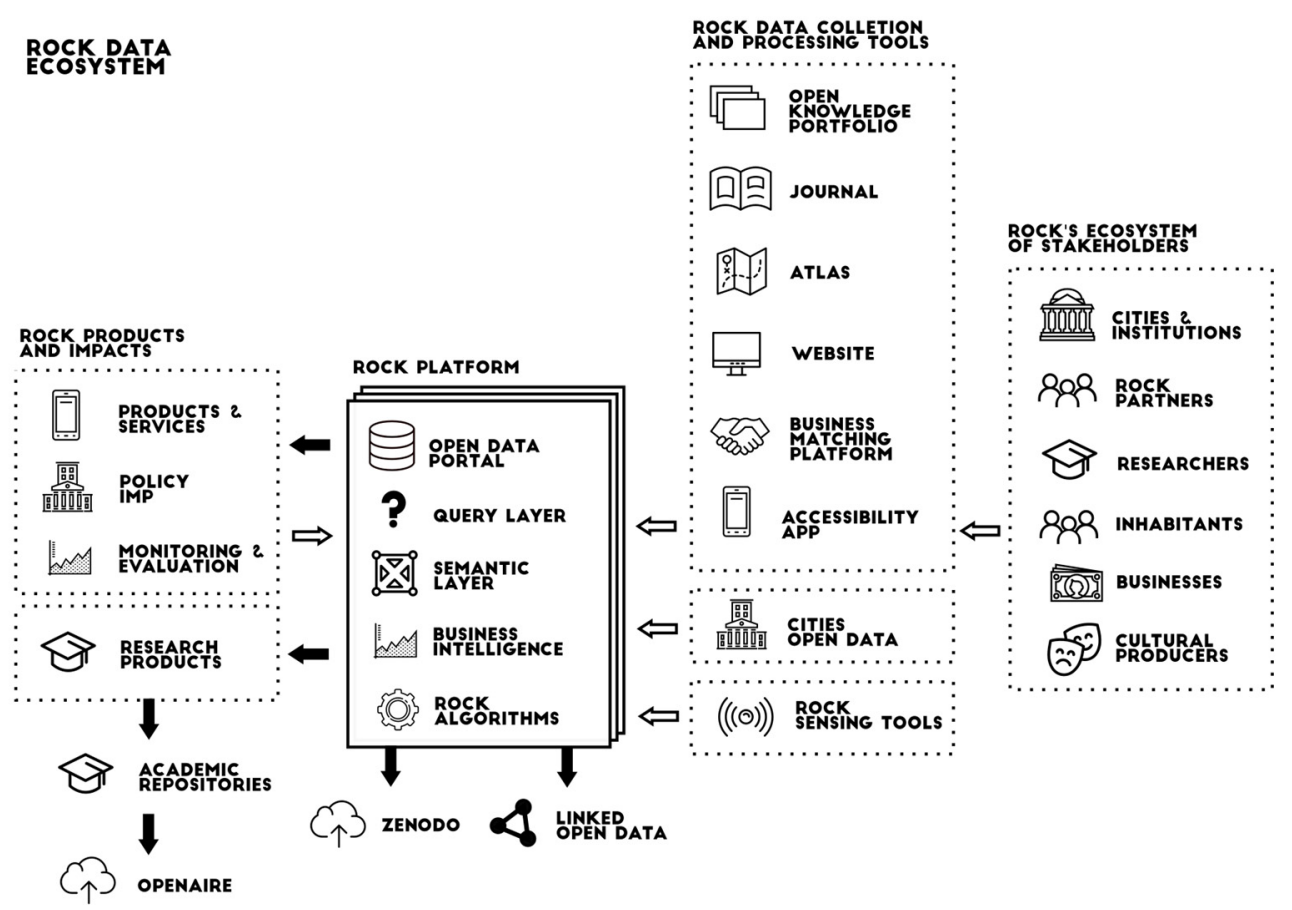

Figure 2: The ROCK tool ecosystem [20].

Unlike the previous ones, the PON IDEHA project (Innovation for Data Elaboration in Heritage Areas, 2017-2021 - No. ARS01_00421) is still under development. The research activity is intended to build, in this case too, an interoperable open source IT platform capable of aggregating different types of data, information and content on $\mathrm{CH}$, integrating sources from different origins (from more traditional repositories to real-time content from IoT sensors deployed on cultural sites, specialized HBIM systems or materials from social 
networks). In addition to a catalogue listing and indexing the resources related to different entities, in different formats and with different content, the expected result is the use of the system for developing data-informed decision support systems for cultural heritage managers, applications and front-end services for navigation and fruition of resources (also immersive and virtual, and on mobile devices), addressed to different users and replicable in a multi-scale way on cultural sites of different size, characteristics and geographical distribution (Fig. 3). These tools enable the development and enhancement of tourism and territories (focusing in particular on Southern Italy), control systems, monitoring and programming of interventions on $\mathrm{CH}$, support to cultural experience and blended training to respond and network institutions, businesses and citizens. According to the requirements of the National Research Plan (NRP) 2014-2020, funding the project, IDEHA defines strict Operational Goals that combine Industrial Research and Experimental Development activities. The multiple functionalities of the IDEHA smart system will be tested and validated on specific sites, identifying the broad concept of $\mathrm{CH}$, including archaeological sites, architectural complexes and farms linked to the production of PGIs [22].

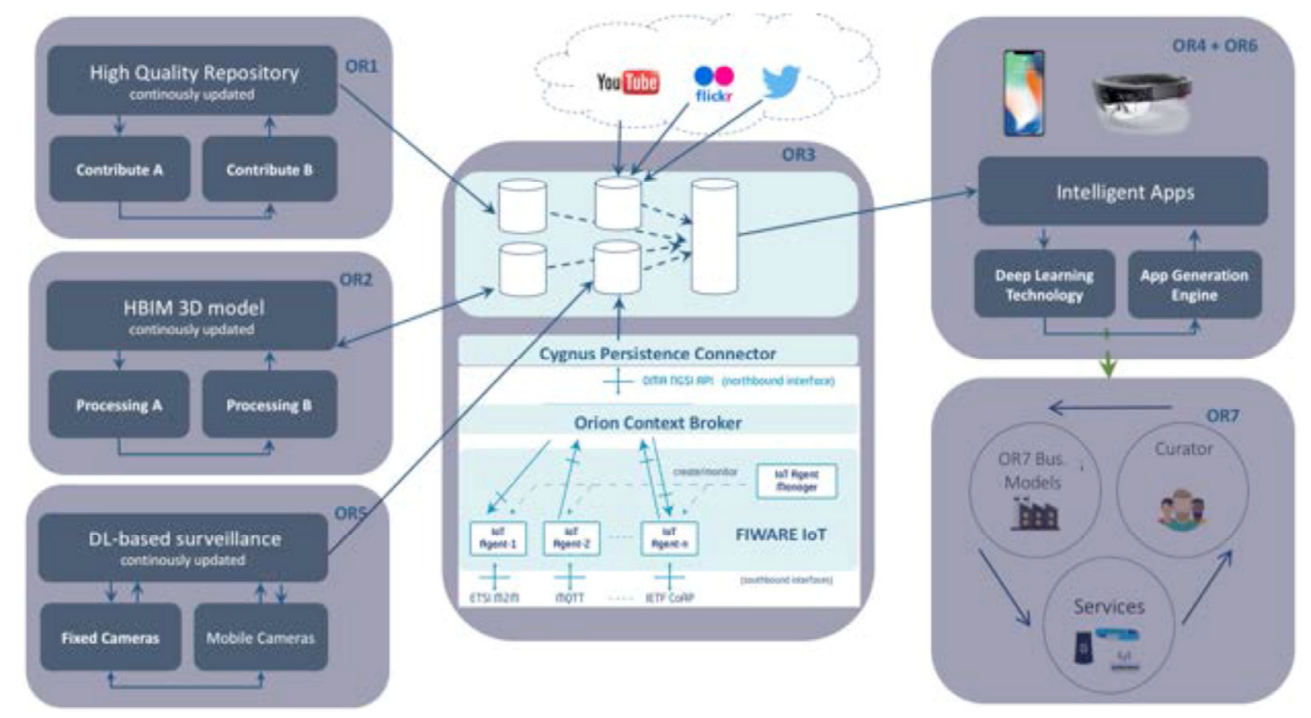

Figure 3: The IDEHA Perth chart [22].

Thus, the three projects prove how the use of the open data interoperable digital platforms is capable to convert the static nature of resources and data into a dynamic and active entity, proving that $\mathrm{CH}$, "if properly managed, can be instrumental in enhancing social inclusion, developing intercultural dialogue, shaping identity of a territory, improving quality of the environment, providing social cohesion and, on the economic side, stimulating tourism development, creating jobs and enhancing investment climate. In other words, investment in heritage can generate return in a form of social benefits and economic growth" [23].

\section{CONCLUSION}

Digital technologies applied to $\mathrm{CH}$ represent an open field of research with a great potentiality. Interoperable platforms are tools to open, connect and use the knowledge heritage linked to the available digital information and data ecosystem. The conservation and 
systematization of this heritage on $\mathrm{CH}$, aside from tackling the overlapping of efforts and the fragmentation of resources, contributes in a holistic and democratic way to the construction of the universal right to knowledge, using it as an active mechanism for access, awareness, valorisation, promotion and conservation of $\mathrm{CH}$. The pandemic emergency linked to the COVID-19 virus made evident how direct access to $\mathrm{CH}$ and the traditional repositories of knowledge (libraries, archives, museums, monumental sites, historical cities, etc.) must necessarily be accompanied and supported, without in any way replacing it, by a prompt and effective digital response. Technological and digital innovation must necessarily interact and be integrated with social and cultural expectations, transformations of contemporary lifestyles and cities according to a holistic, interdisciplinary and sustainable approach. The action and common efforts in fostering $\mathrm{CH}$ must exploit and take full advantage of the possibilities offered by technologies and increase their capabilities by leveraging participation, collaborative interaction and direct connection - which are typical qualities of tools such as the platform - between data/information and users/stakeholders, testing the capacity of the network in new application scenarios (economic, political, social and cultural activities) that were previously disconnected.

In terms of vision, objectives and results achieved, the lessons learned from the INCEPTION, ROCK and IDEHA projects provide a contribute in demonstrating the impacts that an open, interoperable and collaborative digital system can have on different levels (architectural, urban, etc.) involving a wide and diverse range of stakeholders. Beyond the peculiarities of the three projects, their cross-cutting contribution is the demonstration on how to make use of the available information, to produce new ones and through which tools, and to give an open and operable quality to the results achieved in terms of data collection, good practices, approaches and methodologies developed, enabling their use for the construction of new knowledge scenarios.

\section{ACKNOWLEDGEMENTS}

The PON IDEHA project has received funding within the Industrial Research Projects and Experimental Development PNR 2015-2020. No. ARS01_00421. The INCEPTION and ROCK projects are co-financed by the European Union within the H2020 Framework Programme (INCEPTION G.A. No.665220, ROCK G.A. No.730280).

\section{REFERENCES}

[1] Treccani online vocabulary. www.treccani.it/vocabolario/piattaforma/. Accessed on: 10 Jun. 2020.

[2] UNESCO, Convention Concerning The Protection Of The World Cultural And Natural Heritage, Paris, 16 Nov. 1972.

[3] Legislative Decree 22 January 2004, n. 42 - Code of cultural heritage and landscape of the Italian Republic.

[4] ICOMOS, Cultural Heritage, The UN Sustainable Development Goals, and the New Urban Agenda, 15 Feb. 2016.

[5] UNESCO, Convention for the Safeguarding of the Intangible Cultural Heritage, Paris, 17 Oct. 2003.

[6] Council of Europe, Framework Convention on the Value of Cultural Heritage for Society, Faro, 27 Oct. 2005.

[7] Hilbert, M., How much information is there in the "information society"? Significance, 9(8), pp. 8-12, 2012.

[8] UNCTAD, UNCTAD unpacks: How the coronavirus reveals the need to bridge the digital divide, 15 Apr. 2020. 
[9] Kituyi, M., Making digital work for sustainable development: The time to act is now. https://unctad.org/en/pages/newsdetails.aspx? OriginalVersionID=2394. Accessed on: 15 Jun. 2020.

[10] Sola, L., Interview to Cecilia Bonefeld Dahl, General Director of Digital Europe. MosaicoEuropa (Unioncamere Europe), 5(3), pp. 1-2, 2008.

[11] European Commission, Recommendation on the digitisation and online accessibility of cultural material and digital preservation. 27 Oct. 2011 (2011/711/EU).

[12] European Commission, A New European Agenda for Culture (SWD(2018) 167 final), Art. 5.2 - Digital4Culture, p. 8.

[13] European Commission, EU Member States sign up to cooperate on digitising cultural heritage. 9 Apr. 2019. https://ec.europa.eu/digital-single-market/en/news/eu-memberstates-sign-cooperate-digitising-cultural-heritage. Accessed on: 10 Jun. 2020.

[14] European Content in Global Networks Coordination Mechanisms for Digitisation Programmes, The Lund Principles: conclusions of experts meeting, Lund, Sweden, 4 Apr. 2001.

[15] Dynamic Action Plan for the EU co-ordination of digitisation of cultural and scientific content, 15 Nov. 2005.

[16] Addison, A.C., Santana Quintero, M. \& Severo, M., Cultural heritage repositories: Digital archives for conservation and management. Browsing Architecture: Metadata and Beyond, Fraunhofer IRB: Stuttgart, pp. 306-317, 2008.

[17] European Commission, Policies on digital cultural heritage, 2019. https://ec.europa.eu/ digital-single-market/en/digital-cultural-heritage. Accessed on: 10 Jun. 2020.

[18] INCEPTION project. www.inception-project.eu/en, web application www.inceptionhbim.eu/Platform/ and project information on CORDIS portal https://cordis.europa.eu/project/id/665220/it. Accessed on: 12 Jun. 2020.

[19] Di Giulio, R., Maietti, F., Piaia, E., Medici, M., Ferrari, F. \& Turillazzi, B., Integrated data capturing requirements for $3 \mathrm{~d}$ semantic modelling of cultural heritage: The INCEPTION protocol. ISPRS - International Archives of the Photogrammetry, Remote Sensing and Spatial Information Sciences, XLII-2/W3, pp. 251-257, 2017.

[20] ROCK, https://rockproject.eu/, ROCK ATLAS, https://atlas.rockproject.eu/, Rock web platform, https://opendata.rockproject.eu/rock/\#/home. Accessed on: 12 Jun. 2020.

[21] Boeri, A., Gaspari, J., Gianfrate, V., Longo, D. \& Pussetti, C., Il riuso adattivo dei centri storici. Bologna e Lisbona: soluzioni per la rigenerazione urbana. TECHNE Journal of Technology for Architecture and Environment, 12, pp. 230-237, 2016.

[22] PON PNR IDEHA project information. www.unibo.it/it/ricerca/progetti-e-iniziative/ progetti-pon-pnr/ideha. Accessed on: 12 Jun. 2020.

[23] Dümcke, C. \& Gnedovsky, M., The social and economic value of cultural heritage: Literature review (EENC Paper), July 2013. www.eenca.com/index.cfm/publications/ the-social-and-economic-value-of-cultural-heritage-literature-review/. Accessed on: 12 Jun. 2020. 